Editorial

\title{
IoT: A New Open Access Journal for Internet of Things
}

\author{
Marco Picone 1 \\ Department of Sciences and Methods for Engineering (DISMI), University of Modena and Reggio Emilia, \\ Via Amendola 2, Pad. Morselli, 42121 Reggio Emilia, Italy; marco.picone@unimore.it
}

Received: 21 September 2020; Accepted: 22 September 2020; Published: 22 September 2020

During the last decade, the Information Technology ecosystem has been significantly revolutionized by the advent of the Internet of Things (IoT) and its pervasive and quick evolution. It involves billions of interconnected devices denoted as "Smart Objects" able to generate and consume a massive amount of heterogenous data through a multitude of different protocols, technologies, application scenarios and use cases.

The new communication and cyber-physical interaction paradigms (e.g., associated with Digital Twin technologies [1]) together with the massive amount of information being created by the IoT has the power to revolutionize everything from Industry, Smart Cities and Healthcare, allowing them to work more efficiently and profitably, and creating a new class of services and applications. In this challenging context and ecosystem, multiple technologies and research fields emerge as new fundamental pillars for the next generation of IoT developments and innovations.

On one hand, for example, Fog [2] and Edge Computing [3] paradigms started moving services and resources (e.g., storage, processing and networking features) from a centralized vision to the very edge of the network and at the same time, with software-driven networking and microservices-oriented architectures, the operative functionalities are broken down into small, modular, independently deployable and loosely-coupled cooperating functionalities and microelements. This trend is also confirmed and augmented by the next IoT-oriented revolution brought by $5 \mathrm{G}$ networks and Multi-Access Edge Computing (MEC) architectures [4].

On the other hand, Machine Learning and Artificial Intelligence revolutionized, and transformed, IoT [5] research by introducing new advanced possibilities and features by giving to existing and new applications the power to learn and understand from heterogenous information and datasets. Intelligent IoT grew up as the research field merging multiple technologies and skills to support and create cognitive systems able to automatically understand the operational context, adapt, reason and act in real-time according to changes, external inputs or to reach defined goals. The massive volumes of information being created by the IoT has the power to feed new IoT cognitive systems (both in the Cloud and at the Edge) and augment their capabilities to understand the context, discover new behaviors and operate more efficiently and profitably.

These trends and research fields exemplify how interdisciplinary approaches may easily lead to new knowledge, innovative solutions and an effective integration between Internet of Things and different research ecosystems in order to build the new generation of intelligent cyber-physical IoT systems.

The open access journal IoT started targeting these strategic and fundamental topics and, together with an excellent editorial team, my belief is that this new journal will find its specific and remarkable space in the field of Internet of Things. This journal aims to build the interdisciplinary bridge between the multiple natures and peculiarities of the Internet of Things, combined in one journal with the contributions coming from the academic and the industrial research communities. 
This challenging goal will be possible only with the support of every member of our editorial board together with the strategic effort of the editorial support office. The best achievement of the journal will be to become a solid and inspiring reference for the heterogenous IoT community and we highly motivate the authors to submit their contributions in order also to benefit from the effectiveness of the open access spirit to reach a wider audience of researchers, service developers and IoT innovators. We strongly invite researchers in academia and in industry to submit their original contributions and high-quality manuscripts, as well as comprehensive review papers, but also to contribute to this emerging scientific community to innovative IoT technologies and services.

Conflicts of Interest: The author declares no conflict of interest.

\section{References}

1. Minerva, R.; Lee, G.M.; Crespi, N. Digital Twin in the IoT Context: A Survey on Technical Features, Scenarios, and Architectural Models. Available online: https://ieeexplore.ieee.org/document/9120192 (accessed on 21 September 2020).

2. Bonomi, F.; Milito, R.; Zhu, J.; Addepalli, S. Fog Computing and its Role in the Internet of Things. In Proceedings of the MCC Workshop on Mobile Cloud Computing, Helsinki, Finland, 17 August 2012.

3. Yu, W.; Liang, F.; He, X.; Hatcher, W.G.; Lu, C.; Lin, J.; Yang, X. A Survey on the Edge Computing for the Internet of Things. IEEE Access 2018, 6, 6900-6919. [CrossRef]

4. Porambage, P.; Okwuibe, J.; Liyanage, M.; Ylianttila, M.; Taleb, T. Survey on Multi-Access Edge Computing for Internet of Things Realization. IEEE Commun. Surv. Tutor. 2018, 20, 2961-2991. [CrossRef]

5. Li, H.; Ota, K.; Dong, M. Learning IoT in Edge: Deep Learning for the Internet of Things with Edge Computing. IEEE Netw. 2018, 32, 96-101. [CrossRef]

(C) 2020 by the author. Licensee MDPI, Basel, Switzerland. This article is an open access article distributed under the terms and conditions of the Creative Commons Attribution (CC BY) license (http://creativecommons.org/licenses/by/4.0/). 